\title{
Diagonally and antidiagonally symmetric alternating sign matrices of odd order
}

\author{
Roger E. Behrend叫, Ilse Fischer非, and Matjaž Konvalinka非 \\ ${ }^{1}$ School of Mathematics, Cardiff University, Cardiff, CF24 4AG, UK \\ ${ }^{2}$ Fakultät für Mathematik, Universität Wien, Oskar-Morgenstern-Platz 1, 1090 Wien, Austria \\ ${ }^{3}$ Fakulteta za matematiko in fiziko, Univerza v Ljubljani in Inštitut za matematiko, fiziko in mehaniko, Jadranska 19, \\ Ljubljana, Slovenia
}

\begin{abstract}
We study the enumeration of diagonally and antidiagonally symmetric alternating sign matrices (DASASMs) of fixed odd order by introducing a case of the six-vertex model whose configurations are in bijection with such matrices. The model involves a grid graph on a triangle, with bulk and boundary weights which satisfy the YangBaxter and reflection equations. We obtain a general expression for the partition function of this model as a sum of two determinantal terms, and show that at a certain point each of these terms reduces to a Schur function. We are then able to prove a conjecture of Robbins from the mid 1980's that the total number of $(2 n+1) \times(2 n+1)$ DASASMs is $\prod_{i=0}^{n} \frac{(3 i) !}{(n+i) !}$, and a conjecture of Stroganov from 2008 that the ratio between the numbers of $(2 n+1) \times(2 n+1)$ DASASMs with central entry -1 and 1 is $n /(n+1)$. Among the several product formulae for the enumeration of symmetric alternating sign matrices which were conjectured in the 1980's, that for odd-order DASASMs is the last to have been proved.

Résumé. Nous étudions l'énumération de matrices à signes alternants symétriques diagonalement et antidiagonalement (abrégé DASASM) d'ordre impair fixé, en introduisant un cas du modèle à six sommets dont les configurations sont en bijection avec de telles matrices. Le modèle comporte un graphe sur un réseau triangulaire, dont les poids satisfont les équations Yang-Baxter et de réflexion. Nous obtenons une expression générale pour la fonction de partition de ce modèle comme une somme de deux termes déterminantaux, et nous montrons qu' à un certain point chacun de ces termes se réduit à une fonction de Schur. Nous sommes alors en mesure de prouver une conjecture de Robbins du milieu des années 1980 prévoyant que le nombre total de DASASMs d'ordre $(2 n+1) \times(2 n+1)$ est $\prod_{i=0}^{n} \frac{(3 i) !}{(n+i) !}$, et une conjecture de Stroganov de 2008 prévoyant que le rapport entre le nombre de $(2 n+1) \times(2 n+1)$ DASASMs avec coefficient central -1 et 1 est $n /(n+1)$. Parmi les nombreuses formules de produit pour l'énumération des matrices à signe alternant symétriques qui ont été conjecturées dans les années 1980, celle-ci est la derniére à être prouvée.
\end{abstract}

Keywords. Exact enumeration, alternating sign matrices, six-vertex model

\footnotetext{
${ }^{\dagger}$ Email: behrendrecardiff.ac.uk. The first two authors acknowledge hospitality and support from the Galileo Galilei Institute, Florence, Italy, during the programme "Statistical Mechanics, Integrability and Combinatorics" held in May-June 2015.

\#Email: ilse.fischer@univie.ac.at. Partially supported by Austrian Science Foundation FWF, START grant Y463.

${ }^{\S}$ Email: matjaz.konvalinka@fmf.uni-lj.si. Partially supported by Research Programs L1-069 and Z1-5434 of the Slovenian Research Agency.
}

1365-8050 @ 2016 Discrete Mathematics and Theoretical Computer Science (DMTCS), Nancy, France 


\section{Introduction}

An alternating sign matrix (ASM) is a square matrix in which each entry is 0,1 or -1 , and along each row and column the nonzero entries alternate in sign and have a sum of 1 . These matrices were introduced by Mills, Robbins and Rumsey in the early 1980s, accompanied by various conjectures concerning their enumeration [7, Conjs. 1 \& 2], [8, Conjs. 1-7]. Shortly after this, as discussed by Robbins [14, p. 18], [15, p. 2], Richard Stanley made the important suggestion of systematically studying the enumeration of ASMs invariant under the action of subgroups of the symmetry group of a square. This suggestion led to numerous conjectures for the straight and weighted enumeration of such symmetric ASMs, with these conjectures being summarized by Robbins in a preprint written in the mid 1980s, and placed on the arXiv in 2000 [15]. Much of the content of this preprint also appeared in review papers in 1986 and 1991 by Stanley [16] and Robbins [14], and in 1999 in a book by Bressoud [3, pp. 201-202]. In the preprint, simple product formulae (or, more specifically, recursion relations which lead to such formulae) were conjectured for the straight enumeration of several classes of symmetric ASMs, and it was conjectured that no such product formulae exist for the remaining nonempty classes. All of these formulae have since been proved (see Section 1.1 for further details), except a formula that the number of $(2 n+1) \times(2 n+1)$ diagonally and antidiagonally symmetric ASMs (DASASMs) is $\prod_{i=0}^{n} \frac{(3 i) !}{(n+i) !}$. In this extended abstract, we sketch a proof of this last remaining formula, with the details of the proof being given in the full version of this work, [1]. We also present a related result, which was conjectured by Stroganov in 2008 [17, Conj. 2] and which we prove in [1], that the ratio between the numbers of $(2 n+1) \times(2 n+1)$ DASASMs with central entry -1 and 1 is $n /(n+1)$.

\subsection{Symmetry classes of ASMs}

The symmetry group of a square is the dihedral group $D_{4}=\left\{\mathcal{I}, \mathcal{V}, \mathcal{H}, \mathcal{D}, \mathcal{A}, \mathcal{R}_{\pi / 2}, \mathcal{R}_{\pi}, \mathcal{R}_{-\pi / 2}\right\}$, where $\mathcal{I}$ is the identity, $\mathcal{V}, \mathcal{H}, \mathcal{D}$ and $\mathcal{A}$ are reflections in vertical, horizontal, diagonal and antidiagonal axes, respectively, and $\mathcal{R}_{\theta}$ is counterclockwise rotation by $\theta$. The group has a natural action on the set $\operatorname{ASM}(n)$ of $n \times n$ ASMs, in which $\mathcal{I}(A)_{i j}=A_{i j}, \mathcal{V}(A)_{i j}=A_{i, n+1-j}, \mathcal{H}(A)_{i j}=A_{n+1-i, j}, \mathcal{D}(A)_{i j}=A_{j i}, \mathcal{A}(A)_{i j}=$ $A_{n+1-j, n+1-i}, \mathcal{R}_{\pi / 2}(A)_{i j}=A_{j, n+1-i}, \mathcal{R}_{\pi}(A)_{i j}=A_{n+1-i, n+1-j}$ and $\mathcal{R}_{-\pi / 2}(A)_{i j}=A_{n+1-j, i}$, for any $A \in \operatorname{ASM}(n)$. The group has ten subgroups, $\{\mathcal{I}\},\{\mathcal{I}, \mathcal{V}\} \approx\{\mathcal{I}, \mathcal{H}\},\left\{\mathcal{I}, \mathcal{V}, \mathcal{H}, \mathcal{R}_{\pi}\right\},\{\mathcal{I}, \mathcal{D}\} \approx\{\mathcal{I}, \mathcal{A}\}$, $\left\{\mathcal{I}, \mathcal{D}, \mathcal{A}, \mathcal{R}_{\pi}\right\},\left\{\mathcal{I}, \mathcal{R}_{\pi}\right\},\left\{\mathcal{I}, \mathcal{R}_{\pi / 2}, \mathcal{R}_{\pi}, \mathcal{R}_{-\pi / 2}\right\}$ and $D_{4}$, where $\approx$ denotes conjugacy. In studying the enumeration of symmetric ASMs, the primary task is to obtain formulae for the cardinalities of each set $\operatorname{ASM}(n, H)$ of $n \times n$ ASMs invariant under the action of subgroup $H$. Since this cardinality is the same for conjugate subgroups, there are eight classes. The standard choices and names for these classes, together with information about empty subclasses, conjectures and proofs of straight enumeration formulae, and numerical data are as follows.

- $\operatorname{ASM}(n)$. Unrestricted ASMs. A formula was conjectured by Mills, Robbins and Rumsey [7, Conj. 1], and proved by Zeilberger [18, p. 5], with further proofs, involving different methods, subsequently being obtained by Kuperberg [5] and Fischer [4].

- $\operatorname{ASM}(n,\{\mathcal{I}, \mathcal{V}\})$. Vertically symmetric ASMs . For $n$ even, the set is empty. For $n$ odd, a formula was conjectured by Robbins [15, Sec. 4.2], and proved by Kuperberg [6, Thm. 2].

- $\operatorname{ASM}\left(n,\left\{\mathcal{I}, \mathcal{V}, \mathcal{H}, \mathcal{R}_{\pi}\right\}\right)$. Vertically and horizontally symmetric ASMs. For $n$ even, the set is empty. For $n$ odd, formulae were conjectured by Mills [15, Sec. 4.2], and proved by Okada [11, Thm. 1.2(A5)].

- $\operatorname{ASM}\left(n,\left\{\mathcal{I}, \mathcal{R}_{\pi}\right\}\right)$. Half-turn symmetric ASMs. Formulae were conjectured by Mills, Robbins and Rumsey [9, p. 285], and proved for $n$ even by Kuperberg [6, Thm. 2], and $n$ odd by Razumov and 
Stroganov [13, p. 1197].

- $\operatorname{ASM}\left(n,\left\{\mathcal{I}, \mathcal{R}_{\pi / 2}, \mathcal{R}_{\pi}, \mathcal{R}_{-\pi / 2}\right\}\right)$. Quarter-turn symmetric ASMs. For $n \equiv 2(\bmod 4)$, the set is empty. For $n \not \equiv 2(\bmod 4)$, formulae were conjectured by Robbins [15, Sec. 4.2], and proved for $n \equiv 0(\bmod 4)$ by Kuperberg [6, Thm. 2], and $n$ odd by Razumov and Stroganov [12, p. 1649].

- $\operatorname{ASM}(n,\{\mathcal{I}, \mathcal{D}\})$. Diagonally symmetric ASMs. No formula is currently known or conjectured. Data for $n \leq 20$ is given by Bousquet-Mélou and Habsieger [2, Tab. 1].

- $\operatorname{ASM}\left(n,\left\{\mathcal{I}, \mathcal{D}, \mathcal{A}, \mathcal{R}_{\pi}\right\}\right)$. Diagonally and antidiagonally symmetric ASMs. For $n$ even, no formula is currently known or conjectured. Data for $n \leq 24$ is given by Bousquet-Mélou and Habsieger [2, Tab. 1]. For $n$ odd, a formula was conjectured by Robbins [15, Sec. 4.2], and is proved in [1], with a sketch of the proof being given in this extended abstract.

- $\operatorname{ASM}\left(n, D_{4}\right)$. Totally symmetric ASMs. For $n$ even, the set is empty. For $n$ odd, no formula is currently known or conjectured. Data for $n \leq 27$ is given by Bousquet-Mélou and Habsieger [2, Tab. 1].

In addition to the eight standard symmetry classes of ASMs, various closely related classes, most of which were introduced by Kuperberg [6], have also been studied.

\subsection{DASASMs and odd DASASM triangles}

The set $\operatorname{ASM}\left(n,\left\{\mathcal{I}, \mathcal{D}, \mathcal{A}, \mathcal{R}_{\pi}\right\}\right)$ of all $n \times n$ DASASMs will be denoted in the rest of this extended abstract as $\operatorname{DASASM}(n)$, i.e., $\operatorname{DASASM}(n)=\left\{A \in \operatorname{ASM}(n) \mid A_{i j}=A_{j i}=A_{n+1-j, n+1-i}\right\}$.

For example,

$$
\operatorname{DASASM}(3)=\left\{\left(\begin{array}{lll}
1 & 0 & 0 \\
0 & 1 & 0 \\
0 & 0 & 1
\end{array}\right),\left(\begin{array}{ccc}
0 & 1 & 0 \\
1 & -1 & 1 \\
0 & 1 & 0
\end{array}\right),\left(\begin{array}{lll}
0 & 0 & 1 \\
0 & 1 & 0 \\
1 & 0 & 0
\end{array}\right)\right\} .
$$

Consider any $A \in \operatorname{DASASM}(2 n+1)$, or more generally any $(2 n+1) \times(2 n+1)$ half-turn symmetric ASM $A$. The central column (or row) of $A$ is invariant under reversal of the order of its entries, and its nonzero entries alternate in sign and have a sum of 1 . Therefore, the central entry $A_{n+1, n+1}$ is nonzero. The sets of all $(2 n+1) \times(2 n+1)$ DASASMs with fixed central entry 1 and -1 will be denoted as $\operatorname{DASASM}_{+}(2 n+1)$ and DASASM_$(2 n+1)$, respectively

Let an odd DASASM triangle $A$ of order $n$ be a triangular array

$$
\begin{array}{ccccccccc}
A_{11} & A_{12} & A_{13} & \ldots & A_{1, n+1} & \ldots & A_{1,2 n-1} & A_{1,2 n} & A_{1,2 n+1} \\
& A_{22} & A_{23} & \ldots & A_{2, n+1} & \ldots & A_{2,2 n-1} & A_{2,2 n} & \\
& & \ddots & & \vdots & & \ddots & & \\
& & & A_{n n} & A_{n, n+1} & A_{n, n+2} & & & \\
& & & & A_{n+1, n+1}, & & &
\end{array}
$$

such that each entry is 0,1 or -1 and, for each $i=1, \ldots, n+1$, the nonzero entries along the sequence $A_{1 i}, A_{2 i}, \ldots, A_{i-1, i}, A_{i i}, A_{i, i+1}, \ldots, A_{i, 2 n+1-i}, A_{i, 2 n+2-i}, A_{i-1,2 n+2-i}, \ldots, A_{2,2 n+2-i}, A_{1,2 n+2-i}$ (taken to be $A_{1, n+1}, \ldots, A_{n, n+1}, A_{n+1, n+1}, A_{n, n+1}, \ldots, A_{1, n+1}$ for $\left.i=n+1\right)$ alternate in sign and have a sum of 1 .

It can be seen that there is a bijection from $\operatorname{DASASM}(2 n+1)$ to the set of odd DASASM triangles of order $n$, in which the entries $A_{i j}$ of $A \in \operatorname{DASASM}(2 n+1)$ are simply restricted to $i=1, \ldots, n+1$ and $j=i, \ldots, 2 n+2-i$. 
For example, the set of odd DASASM triangles of order 1 is

$$
\left\{\begin{array}{ccccccccc}
1 & 0 & 0 & 0 & 1 & 0 & 0 & 0 & 1 \\
& 1 & , & & -1 & , & & 1 &
\end{array}\right\} .
$$

\subsection{The six-vertex model for odd-order DASASMs}

Define a grid graph on a triangle as

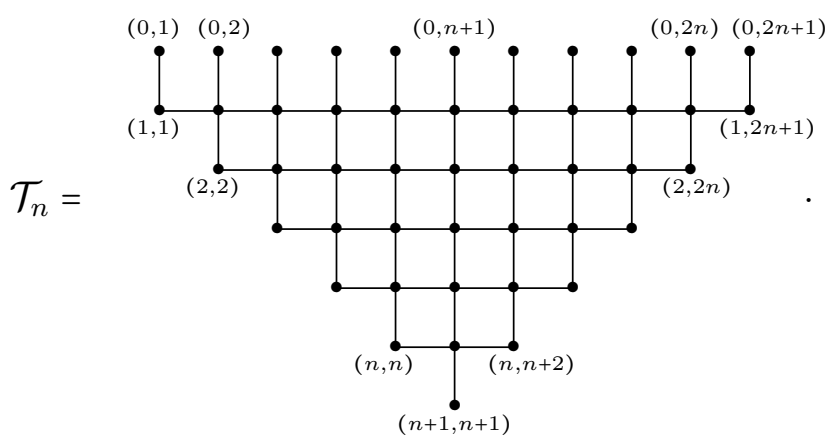

The vertices of $\mathcal{T}_{n}$ consist of top vertices $(0, j), j=0, \ldots, 2 n+1$, of degree 1 , left boundary vertices $(i, i), i=1, \ldots, n$, of degree 2 , bulk vertices $(i, j), i=1, \ldots, n, j=i+1, \ldots, 2 n+1-i$, of degree 4 , right boundary vertices $(i, 2 n+2-i), i=1, \ldots, n$, of degree 2 , and a bottom vertex $(n+1, n+1)$ of degree 1 .

Now define a configuration of the six-vertex model on $\mathcal{T}_{n}$ to be an orientation of the edges of $\mathcal{T}_{n}$, such that each edge incident with a top vertex is directed upwards, and among the four edges incident to any bulk vertex, two are directed into and two are directed out of the vertex, i.e., the so-called six-vertex rule is satisfied.

For example, the set of configurations of the six-vertex model on $\mathcal{T}_{1}$ is

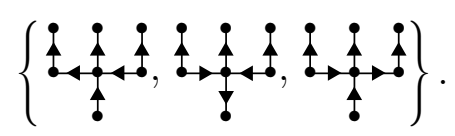

It can be shown straightforwardly that there is a natural bijection from the set of configurations of the six-vertex model on $\mathcal{T}_{n}$ to the set of odd DASASM triangles of order $n$. Essentially, this is a restriction of the standard bijection from the set of configurations of the six-vertex model on an $n \times n$ grid with domain-wall boundary conditions to the set of $n \times n$ ASMs.

For a configuration $C$ of the six-vertex model on $\mathcal{T}_{n}$, and a vertex $(i, j)$ of $\mathcal{T}_{n}$, define the local configuration $C_{i j}$ at $(i, j)$ to be the orientation of the edges incident to $(i, j)$. Hence, the possible local configurations are:

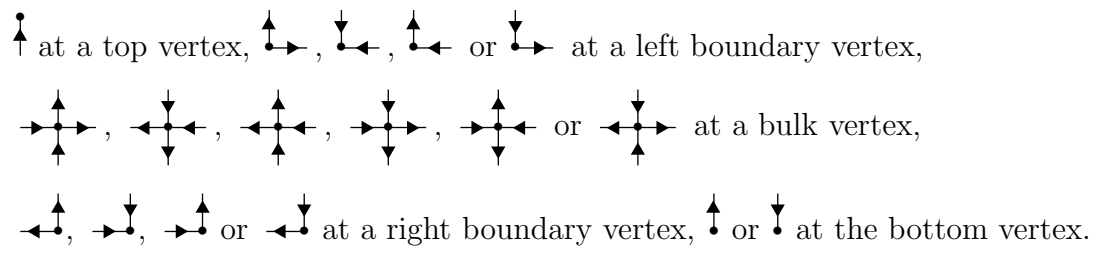


For each possible local configuration $c$ at a bulk or boundary vertex, for an indeterminate $u$, known as a spectral parameter, and for a further indeterminate $q$, assign a weight $W(c, u)$, as given in Table 1 , where throughout the rest of this extended abstract, we will use the notation

$$
\bar{x}=x^{-1} \quad \text { and } \quad \sigma(x)=x-\bar{x} .
$$

\begin{tabular}{|c|c|c|}
\hline Bulk weights & Left boundary weights & Right boundary weights \\
\hline$W(\rightarrow \stackrel{4}{\leftarrow}, u)=W(\leftrightarrow, u)=1$ & \multirow{3}{*}{ 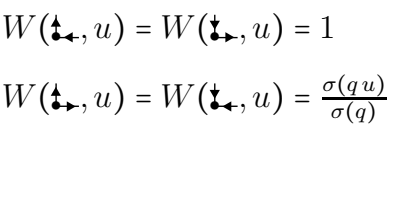 } & \multirow{3}{*}{ 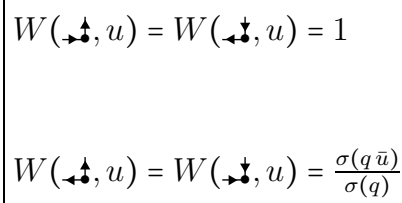 } \\
\hline 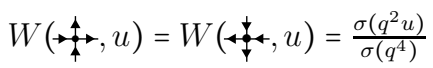 & & \\
\hline$W(\leftrightarrow, u)=W(\nleftarrow, u)=\frac{\sigma\left(q^{2} \bar{u}\right)}{\sigma\left(q^{4}\right)}$ & & \\
\hline
\end{tabular}

Tab. 1: Bulk and boundary weights.

It can be seen that these weights satisfy $\left.W(c, 1)\right|_{q=e^{i \pi / 6}}=1$ for each local configuration $c$ at a bulk vertex, and $W(c, 1)=1$ for each local configuration $c$ at a boundary vertex.

For a configuration $C$ of the six-vertex model on $\mathcal{T}_{n}$, and indeterminates $u_{1}, \ldots, u_{n+1}$, let the weight of left boundary vertex $(i, i)$ be $W\left(C_{i i}, u_{i}\right)$, the weight of bulk vertex $(i, j)$ be $W\left(C_{i j}, u_{i} u_{\min (j, 2 n+2-j)}\right)$, and the weight of right boundary vertex $(i, 2 n+1-i)$ be $W\left(C_{i, 2 n+1-i}, u_{i}\right)$, where, as before, $C_{i j}$ is the local configuration of $C$ at $(i, j)$. Note that $q$ is an overall constant, which is the same in all of these weights.

The assignment of spectral parameters in these weights can be illustrated, for $n=3$, as

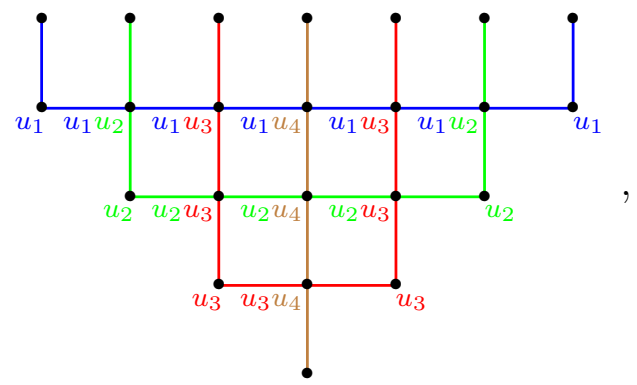

where the color coding indicates that for $i=1, \ldots, n+1, u_{i}$ can be naturally associated with the edges in column $i$, row $i$ and column $2 n+2-i$ of $\mathcal{T}_{n}$, such that the parameter for the weight of a boundary vertex is the single parameter associated with the incident edges, and the parameter for the weight of a bulk vertex is the product of the two different parameters associated with the incident edges.

Now define the odd-order DASASM partition function $Z\left(u_{1}, \ldots, u_{n+1}\right)$ to be the sum of products of bulk and boundary vertex weights, over all configurations $C$ of the six-vertex model on $\mathcal{T}_{n}$. For example, in the case of $\mathcal{T}_{1}$, it follows from (4) that

$$
Z\left(u_{1}, u_{2}\right)=\frac{\sigma\left(q^{2} \bar{u}_{1} \bar{u}_{2}\right) \sigma\left(q \bar{u}_{1}\right)}{\sigma\left(q^{4}\right) \sigma(q)}+\frac{\sigma\left(q u_{1}\right) \sigma\left(q \bar{u}_{1}\right)}{\sigma(q)^{2}}+\frac{\sigma\left(q u_{1}\right) \sigma\left(q^{2} u_{1} u_{2}\right)}{\sigma(q) \sigma\left(q^{4}\right)},
$$


It is clear that

$$
|\operatorname{DASASM}(2 n+1)|=\left.Z(\underbrace{1, \ldots, 1}_{n+1})\right|_{q=e^{i \pi / 6}} .
$$

\section{Main results}

Theorem 1. The odd-order DASASM partition function is given by

$$
\begin{aligned}
& Z\left(u_{1}, \ldots, u_{n+1}\right)= \\
& \frac{\sigma\left(q^{2}\right)^{n}}{\sigma(q)^{2 n} \sigma\left(q^{4}\right)^{n^{2}}} \prod_{i=1}^{n} \frac{\sigma\left(u_{i}\right) \sigma\left(q u_{i}\right) \sigma\left(q \bar{u}_{i}\right) \sigma\left(q^{2} u_{i} u_{n+1}\right) \sigma\left(q^{2} \bar{u}_{i} \bar{u}_{n+1}\right)}{\sigma\left(u_{i} \bar{u}_{n+1}\right)} \prod_{1 \leq i<j \leq n}\left(\frac{\sigma\left(q^{2} u_{i} u_{j}\right) \sigma\left(q^{2} \bar{u}_{i} \bar{u}_{j}\right)}{\sigma\left(u_{i} \bar{u}_{j}\right)}\right)^{2} \\
& \times\left(\operatorname { d e t } _ { 1 \leq i , j \leq n + 1 } \left(\left\{\begin{array}{ll}
\frac{q^{2}+\bar{q}^{2}+u_{i}^{2}+\bar{u}_{j}^{2}}{\sigma\left(q^{2} u_{i} u_{j}\right) \sigma\left(q^{2} \bar{u}_{i} \bar{u}_{j}\right)}, & i \leq n \\
\frac{u_{n+1}-1}{u_{j}^{2}-1}, & i=n+1
\end{array}\right)+\operatorname{det}_{1 \leq i, j \leq n+1}\left(\left\{\begin{array}{ll}
\frac{q^{2}+\bar{q}^{2}+\bar{u}_{i}^{2}+u_{j}^{2}}{\sigma\left(q^{2} u_{i} u_{j}\right) \sigma\left(q^{2} \bar{u}_{i} \bar{u}_{j}\right)}, & i \leq n \\
\frac{\bar{u}_{n+1}-1}{\bar{u}_{j}^{2}-1}, & i=n+1
\end{array}\right)\right) .\right.\right.
\end{aligned}
$$

In the following result, $s_{\lambda}\left(x_{1}, \ldots, x_{k}\right)$ denotes the Schur polynomial indexed by partition $\lambda$, in variables $x_{1}, \ldots, x_{k}$.

Theorem 2. The odd-order DASASM partition function at $q=e^{i \pi / 6}$ is given by

$$
\begin{aligned}
\left.Z\left(u_{1}, \ldots, u_{n+1}\right)\right|_{q=e^{i \pi / 6}}=3^{-n(n-1) / 2} & \left(\frac{u_{n+1}^{n}}{u_{n+1}+1} s_{(n, n-1, n-1, \ldots, 2,2,1,1)}\left(u_{1}^{2}, \bar{u}_{1}^{2}, \ldots, u_{n}^{2}, \bar{u}_{n}^{2}, \bar{u}_{n+1}^{2}\right)\right. \\
& \left.+\frac{\bar{u}_{n+1}^{n}}{\bar{u}_{n+1}+1} s_{(n, n-1, n-1, \ldots, 2,2,1,1)}\left(u_{1}^{2}, \bar{u}_{1}^{2}, \ldots, u_{n}^{2}, \bar{u}_{n}^{2}, u_{n+1}^{2}\right)\right) .
\end{aligned}
$$

The following result includes the product formula conjectured by Robbins [17, Conj. 2] for the number of odd-order DASASMs.

Corollary 3. The number of $(2 n+1) \times(2 n+1)$ DASASMs is given by

$$
\begin{aligned}
|\operatorname{DASASM}(2 n+1)|= & 3^{-n(n-1) / 2} \times(\text { number of semistandard Young tableaux of shape } \\
& (n, n-1, n-1, \ldots, 2,2,1,1) \text { with entries from }\{1, \ldots, 2 n+1\}) \\
= & \prod_{i=0}^{n} \frac{(3 i) !}{(n+i) !} .
\end{aligned}
$$

Proof. The first equality follows by setting $u_{1}=\ldots=u_{n+1}=1$ in (10), and using (8) and the fact that a Schur polynomial evaluated at 1 's is the number of associated semistandard Young tableaux. The second equality (which was previously obtained by Okada [11, Conj. 5.1(2)]) then follows by applying a standard product formula for the number of semistandard Young tableaux of fixed shape and given upper bound on the entries, and simplifying the resulting expression.

The following result was conjectured by Stroganov [17, Conj. 2].

Theorem 4. The numbers of $(2 n+1) \times(2 n+1)$ DASASMs with fixed central entry 1 and -1 satisfy

$$
\frac{\left|\operatorname{DASASM}_{-}(2 n+1)\right|}{\left|\operatorname{DASASM}_{+}(2 n+1)\right|}=\frac{n}{n+1} \text {. }
$$




\section{Proofs}

In this section, proofs of Theorems 1 and 2 are sketched. The proof of Theorem 4 is omitted. Complete proofs of each of these results are given in [1].

\subsection{Local relations for bulk and boundary weights}

The bulk and boundary weights, as given in Table 1, satisfy certain well-known local relations. Those which will be used in this extended abstract are reduction of the bulk weights at $q^{2}$,

$$
W\left(\underset{b}{a \stackrel{d}{b}-c}, q^{2}\right)=\delta_{a \tilde{d}} \delta_{b \tilde{c}},
$$

where $\tilde{a}$ denotes the reversal of edge orientation $a$ and $\delta$ is the Kronecker delta, vertical and horizontal forms of the Yang-Baxter equation (VYBE and HYBE),
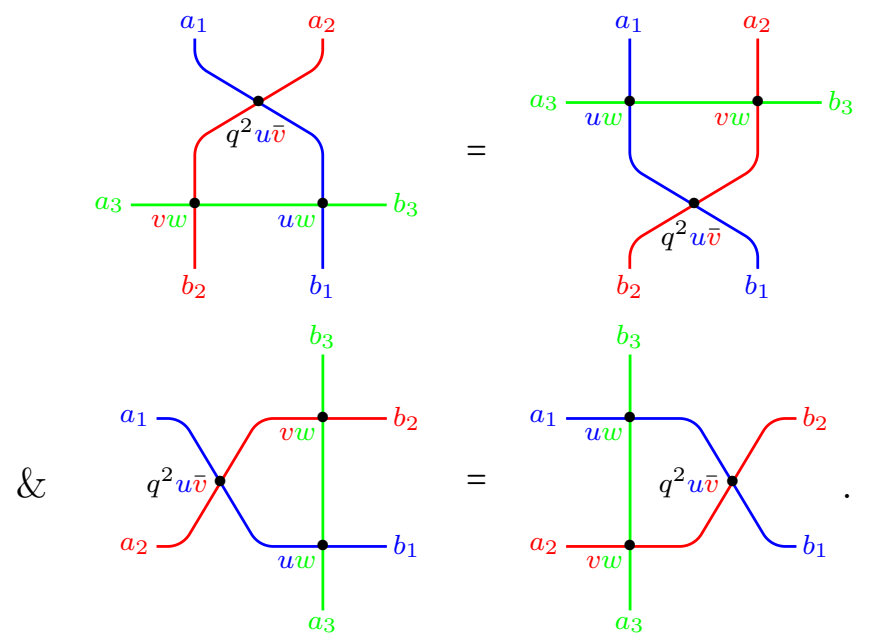

and left and right forms of the reflection (or boundary Yang-Baxter) equation (LRE and RRE),

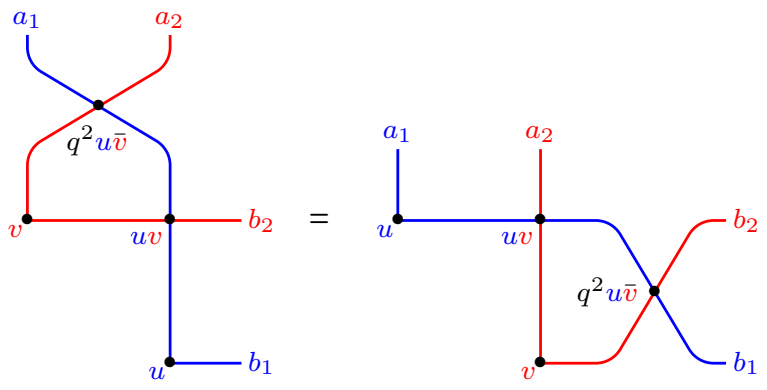




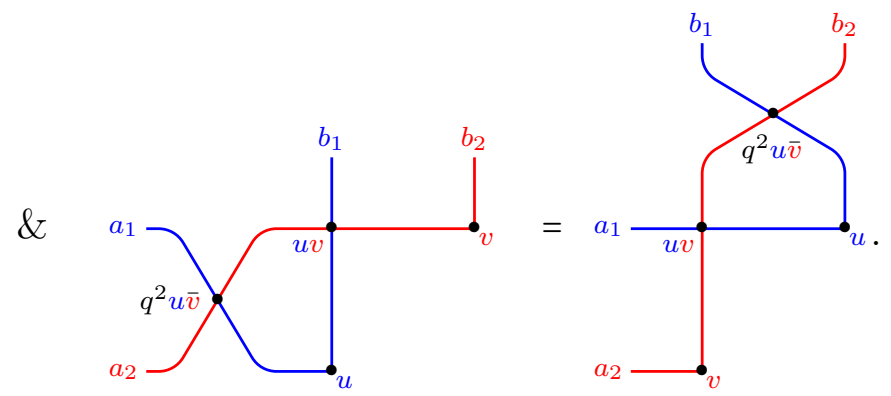

In the Yang-Baxter and reflection equations, we are using standard conventions, which can be summarized as follows. Each graph contains external edges, for which only one of the endpoints is indicated, and internal edges, for which both endpoints are indicated. Each equation holds for all orientations, $a_{1}$, $b_{1}, \ldots$, of the external edges, with an orientation being taken as in or out with respect to the indicated endpoint. For a particular orientation of external edges, each graph denotes a sum, over all orientations of the internal edges which satisfy the six-vertex rule at each degree 4 vertex, of products of weights for each degree 2 and degree 4 vertex shown. If the edges incident to a degree 4 vertex appear horizontally and vertically, with an associated spectral parameter $u$ to the left of and below the vertex, then the weight of the vertex is $W\left(a \stackrel{d}{b}_{b}^{d}, u\right)$, for orientations $a, b, c$ and $d$ of the edges incident left, below, right and above the vertex, respectively. If the edges incident to a degree 4 vertex appear diagonally, then these edges and the associated spectral parameter should be rotated so that the parameter appears to the left of and below the vertex, with the weight then being determined as previously. For the degree 2 vertices, the incident edges always appear in the same configurations as used in the notation for the boundary weights, with the weights being determined accordingly.

For example, the $a_{1}=a_{2}=b_{1}=$ in and $b_{2}=$ out case of the right form of the reflection equation is

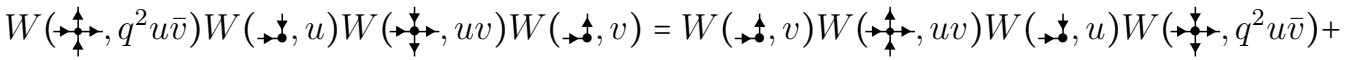

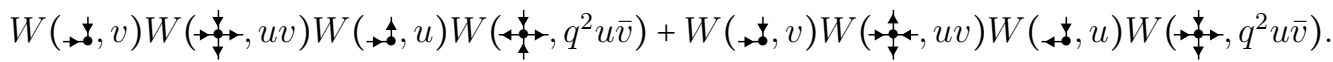

\subsection{Properties of the odd-order DASASM partition function}

Some important properties of the odd-order DASASM partition function will now be identified, together with brief sketches of their proofs.

Proposition 5. The odd-order DASASM partition function satisfies $Z\left(\bar{u}_{1}, \ldots, \bar{u}_{n+1}\right)=Z\left(u_{1}, \ldots, u_{n+1}\right)$.

Proof. This can be proved by reflecting each configuration of the six-vertex model on $\mathcal{T}_{n}$ in the central vertical line of $\mathcal{T}_{n}$, and applying properties of the weights under such reflection.

Proposition 6. The odd-order DASASM partition function $Z\left(u_{1}, \ldots, u_{n+1}\right)$ is a Laurent polynomial in $u_{n+1}$ of lower degree at least $-n$ and upper degree at most $n$, and is even in $u_{i}$, for each $i=1, \ldots, n$.

Proof. This follows straightforwardly from the definitions of the partition function and the weights.

Proposition 7. The odd-order DASASM partition function $Z\left(u_{1}, \ldots, u_{n+1}\right)$ is symmetric in $u_{1}, \ldots, u_{n}$. 
Proof. This can be proved using an approach analogous to that used by Kuperberg [6, Lem. 11 \& Fig. 13] to show that the partition function for $4 n \times 4 n$ off-diagonally off-antidiagonally symmetric ASMs is symmetric in all of its spectral parameters. First note that it is sufficient to show that $Z\left(u_{1}, \ldots, u_{n+1}\right)$ is symmetric in $u_{i}$ and $u_{i+1}$, for $i=1, \ldots, n-1$. The proof of this for $n=3$ and $i=2$ can be illustrated as

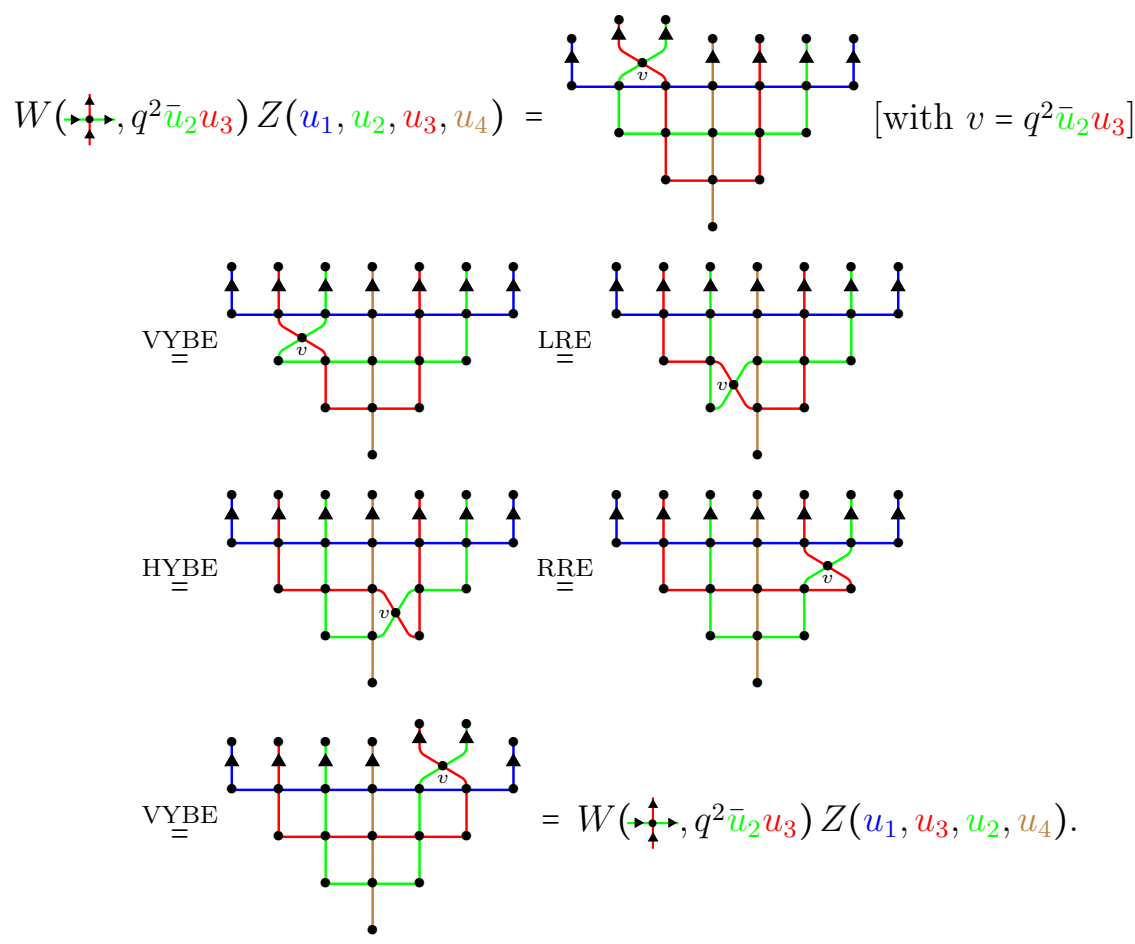

The assignment of spectral parameters and notation here are based on those used in (6) and in Section 3.1 In particular, each graph denotes a sum, over all orientations of the unlabeled edges which satisfy the six-vertex rule at each degree 4 vertex, of products of weights for each degree 2 and degree 4 vertex, with the spectral parameters for unlabeled vertices being determined similarly to the example in (6). The abbreviations above the = signs indicate the local relations from Section 3.1 which give the associated equalities.

Proposition 8. If $u_{1} u_{n+1}=q^{2}$, then the odd-order DASASM partition function satisfies

$$
Z\left(u_{1}, \ldots, u_{n+1}\right)=\frac{\sigma\left(q u_{1}\right)\left(\sigma\left(q \bar{u}_{1}\right)+\sigma(q)\right) \prod_{i=2}^{n} \sigma\left(q^{2} u_{1} u_{i}\right) \sigma\left(q^{2} u_{i} u_{n+1}\right)}{\sigma(q)^{2} \sigma\left(q^{4}\right)^{2 n-2}} Z\left(u_{2}, \ldots, u_{n}, u_{1}\right) .
$$

Proof. The proof for $n=3$ can be illustrated as

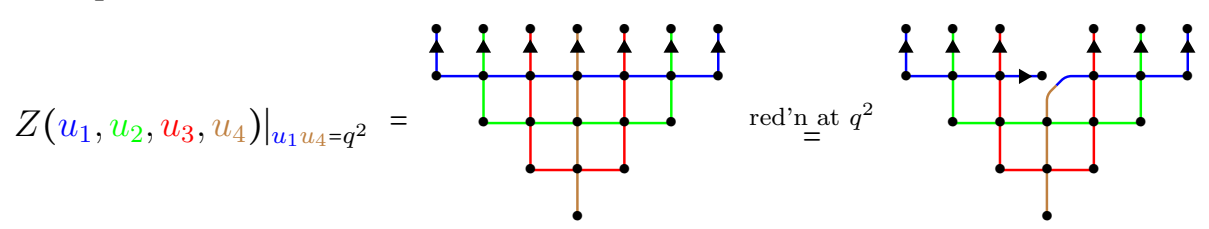




$$
\begin{aligned}
& =\frac{\sigma\left(q u_{1}\right) \sigma\left(q^{2} u_{1} u_{2}\right) \sigma\left(q^{2} u_{1} u_{3}\right)}{\sigma(q) \sigma\left(q^{4}\right)^{2}}: \stackrel{i}{?}: \text { ? } \\
& =\frac{\sigma\left(q u_{1}\right) \sigma\left(q^{2} u_{1} u_{2}\right) \sigma\left(q^{2} u_{1} u_{3}\right)}{\sigma(q) \sigma\left(q^{4}\right)^{2}} \\
& \stackrel{\mathrm{HYBE}, \mathrm{RRE}}{=} \frac{\sigma\left(q u_{1}\right) \sigma\left(q^{2} u_{1} u_{2}\right) \sigma\left(q^{2} u_{1} u_{3}\right)}{\sigma(q) \sigma\left(q^{4}\right)^{2}} \text { : ? ? } \\
& \text { RRE, }=\text { VYBE } \frac{\sigma\left(q u_{1}\right) \sigma\left(q^{2} u_{1} u_{2}\right) \sigma\left(q^{2} u_{1} u_{3}\right)}{\sigma(q) \sigma\left(q^{4}\right)^{2}} \text { ? ? ? ? ? } \\
& =\frac{\sigma\left(q u_{1}\right) \sigma\left(q^{2} u_{1} u_{2}\right) \sigma\left(q^{2} u_{2} u_{4}\right) \sigma\left(q^{2} u_{1} u_{3}\right) \sigma\left(q^{2} u_{3} u_{4}\right)}{\sigma(q) \sigma\left(q^{4}\right)^{4}}: \text { ? ? } \\
& =\frac{\sigma\left(q u_{1}\right) \sigma\left(q^{2} u_{1} u_{2}\right) \sigma\left(q^{2} u_{2} u_{4}\right) \sigma\left(q^{2} u_{1} u_{3}\right) \sigma\left(q^{2} u_{3} u_{4}\right)}{\sigma(q) \sigma\left(q^{4}\right)^{4}}\left(\frac{\sigma\left(q \bar{u}_{1}\right)}{\sigma(q)}+1\right) \\
& \times\left(Z_{-}\left(u_{2}, u_{3}, u_{1}\right)+Z_{+}\left(u_{2}, u_{3}, u_{1}\right)\right) \\
& =\frac{\sigma\left(q u_{1}\right)\left(\sigma\left(q \bar{u}_{1}\right)+\sigma(q)\right) \sigma\left(q^{2} u_{1} u_{2}\right) \sigma\left(q^{2} u_{2} u_{4}\right) \sigma\left(q^{2} u_{1} u_{3}\right) \sigma\left(q^{2} u_{3} u_{4}\right)}{\sigma(q)^{2} \sigma\left(q^{4}\right)^{4}} Z\left(u_{2}, u_{3}, u_{1}\right),
\end{aligned}
$$

where the notation is similar to that used in the proof of Proposition 7 .

\subsection{Proofs of Theorems 1 and 2}

Theorem 1 can now be proved, using results from Section 3.2 .

In particular, it can be shown straightforwardly that a family of functions $X\left(u_{1}, \ldots, u_{n+1}\right)$ is uniquely determined if it satisfies the following properties.

(i) $X\left(u_{1}\right)=1$.

(ii) $X\left(u_{1}, \ldots, u_{n+1}\right)$ is a Laurent polynomial in $u_{n+1}$ of lower degree $\geq-n$ and upper degree $\leq n$. 
(iii) If $u_{1} u_{n+1}=q^{2}$, then

$$
X\left(u_{1}, \ldots, u_{n+1}\right)=\frac{\sigma\left(q u_{1}\right)\left(\sigma\left(q \bar{u}_{1}\right)+\sigma(q)\right) \prod_{i=2}^{n} \sigma\left(q^{2} u_{1} u_{i}\right) \sigma\left(q^{2} u_{i} u_{n+1}\right)}{\sigma(q)^{2} \sigma\left(q^{4}\right)^{2 n-2}} X\left(u_{2}, \ldots, u_{n}, u_{1}\right) .
$$

(iv) $X\left(\bar{u}_{1}, \ldots, \bar{u}_{n+1}\right)=X\left(u_{1}, \ldots, u_{n+1}\right)$.

(v) $X\left(u_{1}, \ldots, u_{n+1}\right)$ is even in $u_{i}$, for each $i=1, \ldots, n$.

(vi) $X\left(u_{1}, \ldots, u_{n+1}\right)$ is symmetric in $u_{1}, \ldots, u_{n}$.

If $X\left(u_{1}, \ldots, u_{n+1}\right)$ is taken to be $Z\left(u_{1}, \ldots, u_{n+1}\right)$, i.e., the LHS of [9], then (i)-(vi) are satisfied, since (i) is trivial, while (ii)-(vi) follow from Propositions 5,8 ,

Also, if $X\left(u_{1}, \ldots, u_{n+1}\right)$ is taken to be the RHS of (9), it can be checked using standard properties of determinants that (i)-(vi) are again satisfied, from which the required equality in (9) then follows.

Theorem 2 can be proved by setting $q=e^{i \pi / 6}$ in $[9]$, and then applying a general identity of Okada [10, Thm. 4.2],

$$
\operatorname{det}_{1 \leq i, j \leq k}\left(\frac{a_{i}-b_{j}}{x_{i}-y_{j}}\right)=\frac{(-1)^{k(k+1) / 2}}{\prod_{i, j=1}^{k}\left(x_{i}-y_{j}\right)} \operatorname{det}\left(\begin{array}{ccccccc}
1 & a_{1} & x_{1} & a_{1} x_{1} & \ldots & x_{1}^{k-1} & a_{1} x_{1}^{k-1} \\
1 & b_{1} & y_{1} & b_{1} y_{1} & \ldots & y_{1}^{k-1} & b_{1} y_{1}^{k-1} \\
\vdots & \vdots & \vdots & \vdots & \ddots & \vdots & \vdots \\
1 & a_{k} & x_{k} & a_{k} x_{k} & \ldots & x_{k}^{k-1} & a_{k} x_{k}^{k-1} \\
1 & b_{k} & y_{k} & b_{k} y_{k} & \ldots & y_{k}^{k-1} & b_{k} y_{k}^{k-1}
\end{array}\right) \text {, }
$$

with

$$
a_{i}=\left\{\begin{array}{ll}
u_{i}^{2}+u_{i}^{4}, & i \leq n, \\
-1, & i=n+1,
\end{array} \quad x_{i}=\left\{\begin{array}{ll}
u_{i}^{6}, & i \leq n, \\
1, & i=n+1,
\end{array} \quad b_{j}=\bar{u}_{j}^{2}+\bar{u}_{j}^{4}, \quad y_{j}=\bar{u}_{j}^{6}, \quad k=n+1,\right.\right.
$$

to the first determinant on the RHS of (9). This enables the determinant to be transformed, up to a factor, to a determinant which corresponds to the first Schur polynomial on the RHS of (10). The required result then follows by simplifying various prefactors, and noting that the two terms on the RHS of (9) or (10) are related by replacement of each spectral parameter by its reciprocal.

\section{Acknowledgements}

We thank Arvind Ayyer for very helpful discussions.

\section{References}

[1] R. Behrend, I. Fischer, And M. Konvalinka, Diagonally and antidiagonally symmetric alternating sign matrices of odd order, 2015. arXiv:1512.06030.

[2] M. Bousquet-MÉlou And L. Habsieger, Sur les matrices à signes alternants, Discrete Math., 139 (1995), pp. 57-72. Formal power series and algebraic combinatorics (Montreal, QC, 1992).

[3] D. BRESSOUD, Proofs and confirmations. The story of the alternating sign matrix conjecture, MAA Spectrum, Mathematical Association of America and Cambridge University Press, Washington, DC and Cambridge, 1999. 
[4] I. FISCHER, A new proof of the refined alternating sign matrix theorem, J. Combin. Theory Ser. A, 114 (2007), pp. 253-264. arXiv:math/0507270.

[5] G. KUPERBERG, Another proof of the alternating-sign matrix conjecture, Internat. Math. Res. Notices, (1996), pp. 139-150. arXiv:math/9712207.

[6] - Symmetry classes of alternating-sign matrices under one roof, Ann. of Math. (2), 156 (2002), pp. 835-866. arXiv:math/0008184.

[7] W. Mills, D. Robbins, And H. Rumsey, JR., Proof of the Macdonald conjecture, Invent. Math., 66 (1982), pp. 73-87.

[8] _ Alternating sign matrices and descending plane partitions, J. Combin. Theory Ser. A, 34 (1983), pp. 340-359.

[9] _ Self-complementary totally symmetric plane partitions, J. Combin. Theory Ser. A, 42 (1986), pp. 277-292.

[10] S. OKADA, Applications of minor summation formulas to rectangular-shaped representations of classical groups, J. Algebra, 205 (1998), pp. 337-367.

[11] _ Enumeration of symmetry classes of alternating sign matrices and characters of classical groups, J. Algebraic Combin., 23 (2006), pp. 43-69. arXiv:math/0408234.

[12] A. RaZumov AND Y. STROganov, Enumeration of quarter-turn symmetric alternating-sign matrices of odd-order, Theoret. and Math. Phys., 149 (2006), pp. 1639-1650. arXiv:math-ph/0507003.

[13] — Enumerations of half-turn-symmetric alternating-sign matrices of odd-order, Theoret. and Math. Phys., 148 (2006), pp. 1174-1198. arXiv:math-ph/0504022.

[14] D. RoB BIns, The story of $1,2,7,42,429,7436, \ldots$, Math. Intelligencer, 13 (1991), pp. 12-19.

[15] _ - Symmetry classes of alternating sign matrices, 2000. arXiv:math/0008045.

[16] R. Stanley, A baker's dozen of conjectures concerning plane partitions, in Combinatoire énumérative (Montreal, QC, 1985), vol. 1234 of Lecture Notes in Math., Springer, Berlin, 1986, pp. 285-293.

[17] Y. STROGANOV, 1/N phenomenon for some symmetry classes of the odd alternating sign matrices, 2008. arXiv:0807.2520.

[18] D. Zeilberger, Proof of the alternating sign matrix conjecture, Electron. J. Combin., 3 (1996). Research Paper 13, 84 pp. arXiv:math/9407211. 